\title{
Simplified thermo-elastic modeling of high-performance brakes
}

Prof. Dr. Giampiero Mastinu, Prof. M. Gobbi, Department of Mechanical Engineering, Politecnico di Milano, Italy; C. Cantoni, R. Passoni, Brembo S.p.A., Italy

This manuscript is not available according to publishing restriction. Thank you for your understanding. 